\title{
Risco de integridade da pele prejudicada: avaliação e conduta de enfermagem frente às úlceras por pressão (UPP) em pacientes em terapia intensiva*
}

\author{
Integrity risk of impaired skin: evaluation \\ and management of the nursing to pressure \\ ulcers $(P U)$ in patients in intensive care
}

Rafael Gomes de Sousa' Allison Barros Santana ${ }^{2}$
Recebido em: 12/01/2016.

Aprovado em: 31/10/2016.

1 Enfermeiro. Especialista em enfermagem em cuidados intensivos, PUC-GO, Goiânia, Brasil. E-mail: rafael-pa@hotmail.com.br.

2 Enfermeiro. Professor assistente, faculdade de enfermagem, UFT. Mestrando em Ciências da Saúde, UFT. Especialista em Unidade de Terapia Intensiva, PUC-GO, Palmas, Brasil.

\section{Resumo}

$\mathrm{O}$ trabalho buscou identificar o instrumento mais apropriado para avaliação do risco de úlcera por pressão (UPP) nos pacientes em terapia intensiva. Trata-se de uma revisão integrativa da literatura. Foram selecionados artigos primários, disponíveis na íntegra, nos idiomas português, inglês e espanhol no período de 2000 a 2014, obtidos nas bases de dados ScIELO, MEDLINE, LILACS e IBECS, utilizando os DeCS "úlcera por pressão" and "unidades de terapia intensiva" and "fator de risco". Aplicou-se o teste Critical Appraisal Skills Programme (CASP) para avaliar a qualidade metodológica, sendo os artigos selecionados classificados quanto ao nível de evidência, segundo Scottish Intercollegiate Guidelines Network (SIGN). Obtiveram-se 10 artigos, todos apresentando nível de evidência quatro com boa qualidade metodológica e viés reduzido. Diante dos resultados encontrados, sugere-se a necessidade de novos estudos que correlacione as diversas escalas de avaliação de risco para UPP em pacientes críticos, para que se possa determinar com precisão o melhor instrumento para avaliação dessas feridas.

Palavras-chave: Úlcera por pressão. Unidade de Terapia Intensiva. Fator de riso. Enfermagem.

\begin{abstract}
The research search to identify the most appropriate instrument for assessing the risk of pressure ulcers (PU) in patients in intensive care. It is an integrative literature review. They were selected primary articles, available in full, in portuguese, english and spanish from 2000 to 2014, obtained in databases, ScIELO, MEDLINE, LILACS and IBECS, using the DeCS "pressure ulcer" and "intensive care unit" and "risk factor". Was applied to the test Critical Appraisal Skills Programme (CASP) to assess the methodological quality, and the selected articles classified according to level of evidence, according Scottish Intercollegiate Guidelines Network (SIGN). There was obtained 10 articles, all presenting evidence level four with good methodological quality and reduced bias. Considering the results, It suggests the need for further studies that correlate the different risk assessment scales for PU in critically ill patients, so you can accurately determine the best instrument for evaluation of these wounds.
\end{abstract}

Keywords: Pressure ulcer. Intensive Care Unit. Risk factors. Nursing. 


\section{Introdução}

Define-se úlcera por pressão (UPP) como lesão tecidual, causada por pressão prolongada ou essa em combinação com fricção e cisalhamento (IHI, 2011; NPUAP, 2011). Essas feridas são responsáveis pelo aumento dos custos dispensados a assistência, pois requer tratamento minucioso, alta demanda de tempo, recursos materiais e humanos, prolongando o tempo de internação, elevando a incidência de infecções e a mortalidade (LYRA et al., 2011).

Em estudo descritivo, realizado no Vale do Paraíba, foram avaliados 11 pacientes portadores de UPP encontrando-se um gasto anual de $\mathrm{R} \$ 4.370,16$, sendo $\mathrm{R} \$$ $1.016,56$ com materiais correlatos e $\mathrm{R} \$ 3.353,80$ com coberturas (SIMOES et al., 2010). Por outro lado, as medidas preventivas podem representar uma redução de 25 a 50\% no risco de lesões (ROCHA; MIRANDA; ANDRADE, 2006; CARVALHO, et al., 2007), bem como diminuição de 45\% nos gastos hospitalares (LIMA; GUERRA, 2011).

Vários fatores têm sido associados ao desenvolvimento de UPP. Essas lesões são resultados da conjugação de fatores externos (pressão, cisalhamento e fricção) com fatores internos (idade, sexo, estado nutricional, entre outros) (FERNANDES, 2005). Pacientes em cuidados intensivos estão mais susceptíveis ao risco pela diminuição da mobilidade e sensibilidade, sendo a incidência mais elevada nos indivíduos em pós-operatório e/ou com morbidades respiratória, metabólica, neurológica e/ou infecciosa (GOMES et al., 2010).

Destaca-se que o risco para UPP pode ser avaliado por meio de escalas. O instrumento de avaliação mais extensivamente testado e utilizado é a escala de Braden, embora não tenha sido desenvolvida especificamente para pacientes criticamente enfermos. Validada no Brasil em 1999 possui 6 subescalas, pontuadas com escore entre 6 e 23, usadas largamente nos setores de terapia intensiva devido seu valor preditivo elevado para essa população (CREMASCO et al., 2009; SERPA et al., 2011).

Algumas escalas avaliaram à associação entre UPP e a gravidade do paciente crítico (CREMASCO et al., 2009; SHAHIN; DASSEN; HALFENS, 2009; YEPES et al., 2009). Os escores obtidos na escala de gravidade Acute Physiology And Chronic Health Evaluation (APACHE II), variaram entre 16,6 a 20,4, demonstrando refletir o risco de UPP quando comparada a de Braden. (SHAHIN;
Em estudo realizado com a escala de gravidade Simplified Acute Physiology Score (SAPS II), obtiveram-se 45,87 pontos para os pacientes com UPP e 38,37 para os pacientes sem úlceras (CREMASCO et al., 2009). O resultado indica que quanto maior a gravidade do estado do paciente maior a incidência de UPP.

Nesse sentido, tem se buscando avaliar o risco para o desenvolvimento das UPP com vistas na prevenção e intervenção precoce. Logo, esse estudo propõe identificar na literatura qual instrumento é apropriado na avaliação do risco para UPP em pacientes em terapia intensiva, bem como o papel do enfermeiro na prevenção dessas feridas.

\section{Metodologia}

No presente estudo, selecionou-se como método um dos recursos da prática baseada em evidências (PBE), ou seja, a revisão integrativa da literatura, a qual permite superar estudos primários, abrangendo outras dimensões, possibilitando o estabelecimento de novas teorias e questionamentos (SOARES et al., 2014). A construção dessa revisão foi fundamentada nos estudos que detalham esse método (SOUSA; SILVA; CARVALHO, 2010; SOARES et al., 2014).

$\mathrm{Na}$ presente revisão, as seguintes etapas foram percorridas: estabelecimento da questão norteadora; amostragem na literatura; coleta de dados; análise dos estudos; discussão dos resultados e apresentação da revisão (SOUSA; SILVA; CARVALHO, 2010). Para conduzir o estudo, formulou-se a seguinte questão: qual instrumento é capaz de predizer o risco para úlcera por pressão em pacientes em terapia intensiva?

Os artigos foram obtidos nas bases de dados eletrônico Índice Bibliográfico Espanhol de Ciências de Saúde (IBECS), Scientific Eletronic Library Online (SciELO), Medical Literature Analysis and RetrievalSistem on-line (MEDLINE) e Literatura Latino-Americana e do Caribe em Ciências da Saúde (LILACS). Foram utilizados os seguintes Descritores em Ciências da Saúde (DeCS) e seus correspondentes nas línguas inglesa e espanhola: "Úlcera por pressão" AND "Unidades de Terapia Intensiva” AND "Fator de Risco".

Foram incluídos artigos primários, disponíveis na íntegra nos idiomas português, inglês e espanhol no período de 2000 a 2014, bem como estudos que focalizassem os objetivos e a questão norteadora dessa revisão. Logo, 
foi empregado teste de relevância ao título e ao resumo dos artigos (Apêndice A). Após aceitação preliminar, foi aplicado novo teste (Apêndice B) a metodologia para composição da amostra parcial desse estudo.

A coleta de dados foi realizada em duas etapas, a saber: nos meses de outubro, novembro e dezembro de 2014 foram revisados artigos publicados no período de 2000 a 2013; no mês de Janeiro de 2015 o estudo foi complementado com nova revisão, englobando as publicações do ano anterior.

Aos estudos incluídos aplicou-se o instrumento adaptado Critical Appraisal Skills Programme (CASP) (Anexo A) para avaliar sua qualidade metodológica (MILTON, 2002). Esse instrumento é composto por 10 itens pontuáveis, totalizando 10 pontos, abrangendo: 1) Objetivo do estudo, 2) Adequação do desenho metodológico à questão de estudo, 3) Justificativa dos procedimentos metodológicos, 4) Critérios de seleção da amostra, 5) Detalhamento da coleta de dados, 6) Relação entre pesquisador e pesquisados, 7) Considerações sobre aspectos éticos, 8) Rigor na análise dos dados, 9) Propriedade na apresentação e discussão dos resultados 10) Valor da pesquisa: nota de contribuições, limitações e necessidades de novas pesquisas.

Posteriormente os estudos foram classificados em duas categorias de acordo com a pontuação obtida pela aplicação do instrumento: A) seis a 10 pontos - Estudos de boa qualidade metodológica e viés reduzido e, B) no mínimo cinco pontos - Estudos com qualidade metodológica satisfatória, mas com potencial de viés aumentado.

Consecutivamente, buscou-se colocar em níveis hierárquicos os estudos encontrados, a fim de avaliar criticamente os artigos selecionados com base na força de evidência encontrada, segundo classificação Scottish Intercollegiate Guidelines Network (SIGN) em seis níveis, ou seja, 1. Revisões sistemáticas e meta-análises de ensaios clínicos randomizados 2. Ensaios clínicos randomizados, 3. Estudos não randomizados, 4. Estudos observacionais e 5.Estudos não experimentais e 6. Opinião de experts.

Para a análise e posterior síntese dos artigos que atenderam aos critérios de inclusão, foi utilizado um quadro sinóptico, que contemplou os seguintes aspectos: base de dados, artigos elegíveis, qualidade metodológica e nível de evidência (Quadro 1).

A apresentação dos resultados e discussão dos dados foi realizada de forma descritiva, possibilitando ao leitor avaliar aplicabilidade dos instrumentos, das ações preventivas e de controle realizadas pelo enfermeiro, impactando positivamente na produção de evidências que fundamentem a prática clínica de enfermagem.

\section{Resultados}

Foram encontrados sessenta e sete artigos. Desses, quarenta e três não estavam disponíveis na íntegra, oito não respondiam a questão norteadora e quatro tratavam-se de artigos secundários. Eliminaram-se, ainda, dois artigos, um por estar em idioma diferente do elegível nessa revisão, já outro por se tratar de dissertação. A amostra final foi composta por 10 artigos.

De acordo com a qualidade metodológica, todos os artigos apresentavam conceito A. Conforme o nível de evidência, os artigos foram classificados como categoria 4 , isto é, estudos observacionais, compostos nessa revisão por estudos prospectivos, retrospectivos, caso controle e coorte.

Seis artigos estavam no idioma português, três em inglês e um em espanhol. Seis artigos foram encontrados na base de dados SciELO, três Medline e um LILACS, todos selecionados na primeira etapa da coleta de dados. No segundo cruzamento dos descritores, não foram encontrados outros trabalhos que contemplasse os critérios de inclusão e exclusão, os objetivos e a questão norteadora. Não foram encontrados artigos na base de dados IBECS (Quadro 1).

Quadro 1 - Caracterização dos artigos incluídos na revisão integrativa, janeiro de 2000 a Dezembro de 2014

\begin{tabular}{|lccc|}
\hline Base de dados & Artigos elegíveis & $\begin{array}{c}\text { Qualidade } \\
\text { Metodológica }\end{array}$ & Nível de evidência \\
\hline SciELO & 6 & A & 4 \\
Medline & 3 & A & 4 \\
LILACS & 1 & A & 4 \\
Total & 10 & - & - \\
\hline
\end{tabular}

Legenda: Nível A, 6 a 10 pontos, estudos com boa qualidade metodológica. Categoria 4, estudos observacionais.

Fonte: Autores do estudo.

\section{Discussão}

Como parte do cuidado integral, as pacientes em risco de integridade da pele prejudicada têm empregado escalas para avaliação do risco para UPP (GOMES et al., 2010; SERPA et al., 2011). Atualmente, se reconhece a 
existência de mais de 40 escalas, no entanto, poucas vêm sendo estudadas e utilizadas na prática diária com grupos específicos, como o paciente sujeito a cuidados intensivos (SERPA et al., 2011).

Foram identificadas, nos dez artigos, três escalas estudadas mais extensivamente e comparativamente entre si, sendo elas as escalas de Braden, Norton e Warterlow (GOMES et al., 2010; HINOJOSA; MOREIRA; GUERRA, 2010; ARAUJO et al., 2011; SERPA et al., 2011; ROGENSKI; KURCGANT, 2012a; HYUN et al., 2013; SIMÃO; CALIRI; SANTOS, 2013). Todos os trabalhos apresentaram nível de evidência quatro com boa qualidade metodológica e viés reduzido.

Dentre os artigos selecionados, seis descrevem características da escala de Braden. Esse instrumento de avaliação de risco foi criado nos Estados Unidos em 1987 e validado no Brasil em 1999. Possui seis subescalas: percepção sensorial, atividade, mobilidade, umidade, nutrição, fricção/cisalhamento. Essas subescalas são pontuadas de um a quatro, exceto fricção/cisalhamento, cujo valor varia de um a três. Os escores totais variam de 6 a 23, sendo os índices elevados correspondentes a baixo risco e índices baixos maiores riscos (HINOJOSA; MOREIRA; GUERRA, 2010; COX, 2011; SERPA et al., 2011; ROGENSKI; KURCGANT, 2012a; HYUN et al., 2013; SIMÃO; CALIRI; SANTOS, 2013).

Os pacientes avaliados por meio da escala de Braden são classificados em risco muito alto (escores igual ou menor a 9), risco alto (escores de 10 a 12), risco moderado (escores de 13 a 14), baixo risco (escores de 15 a 18) e sem risco (escores de 19 a 23) (COX, 2011; SERPA et al., 2011; ROGENSKI; KURCGANT, 2012a; SIMÃO; CALIRI; SANTOS, 2013).

Determinou-se nessa escala o escore de corte 18, embora existam controvérsias quanto à relação custo/ benefício da aplicação de medidas preventivas a partir dessa pontuação, uma vez que esse escore apresentou baixo valor preditivo positivo em alguns estudos, variando conforme a população estudada (COX, 2011; SERPA et al., 2011). Identificou-se um bom valor preditivo positivo a partir da pontuação 13, obtidos na terceira avaliação em pacientes em terapia intensiva (SERPA et al., 2011).

Em dois trabalhos as subescalas de Braden percepção sensorial, mobilidade e umidade, foram fatores estaticamente significantes para o desenvolvimento e agravamento da UP em pacientes críticos (ROGENSKI;
KURCGANT, 2012a; SOOKYUNG et al., 2013). A subescala fricção/cisalhamento aumentou em até seis vezes o risco de UP grau II nessa população (COX, 2011).

Em dois artigos, os autores discorrem, brevemente, sobre escala de Norton. Nessa ferramenta são avaliados cinco parâmetros para o risco de UP, sendo eles: condição física, nível de consciência, atividade, mobilidade e incontinência. Cada subescala e pontuada de um a quatro, variando os escores totais entre 5 a 20. Pontuação menor ou igual a 14, paciente em risco. Valores inferiores a 12, alto risco (HINOJOSA; MOREIRA; GUERRA, 2010; ARAUJO; ARAUJO; CAETANO, 2011).

A escala de Wartelow foi desenvolvida, inicialmente, na Inglaterra em 1985 e possui sete tópicos principais: relação peso/altura, avaliação visual da pele em áreas de risco, sexo/idade, continência, mobilidade, apetite e medicações. Possui, também, quatro itens que pontuam fatores de risco especiais: subnutrição do tecido celular, deficit neurológico, tempo de cirurgia acima de duas horas e trauma abaixo da medula lombar. Os pacientes são classificados em risco (10 a14), alto risco (15 a 19) e altíssimo risco para UP (>20) (HINOJOSA; MOREIRA; GUERRA, 2010; ARAUJO; ARAUJO; CAETANO, 2011).

Nessa revisão foi encontrado, apenas, um artigo que avaliou comparativamente as escalas de Braden, Norton e Wartelow. Os autores descobriram uma correlação direta entre as escalas de Norton e Braden, bem como um melhor desempenho da escala de Wartelow sobre as demais, quando aplicada em uma população de adultos jovens do sexo masculino (ARAUJO; ARAUJO; CAETANO, 2011).

Com relação ao papel da equipe de enfermagem na avaliação e prevenção das UPP, foram selecionados oito artigos em que os autores abordam essa temática (BAUMGARTEN et al., 2008; GOMES et al., 2010; HINOJOSA; MOREIRA; GUERRA, 2010; COX, 2011; ROGENSKI; KURCGANT, 2012a; ROGENSKI; KURCGANT, 2012b; HYUN et al., 2013; SIMÃO; CALIRI; SANTOS, 2013). A maioria dos trabalhos recomenda a adoção de escalas de avaliação de risco, em que são identificados os pacientes e os principais fatores predisponentes para o surgimento dessas lesões.

Com base na literatura, destacam-se algumas recomendações na utilização desses instrumentos: avaliação na admissão, diariamente e/ou quando as condições 
clínicas justificarem e treinamento sistematizado de enfermeiros, diminuindo as diferenças interobservadores no momento de aplicação das escalas de avaliação (GOMES et al., 2010; ROGENSKI; KURCGANT, 2012b; SIMÃO; CALIRI; SANTOS, 2013).

Encontramos descritas algumas intervenções preventivas, principalmente executadas pela equipe de enfermagem, envolvendo o uso de colchões adequados ao alívio da pressão e mudanças de decúbito, embora ocorra risco de instabilidade hemodinâmica no paciente crítico (BAUMGARTEN et al., 2008; ROGENSKI; KURCGANT, 2012b; HYUN et al., 2013). Deve ser adotada por toda a equipe de saúde uma maior vigilância na primeira semana de internação, momento de maior alteração fisiológica do paciente e com probabilidade elevada de lesões (COX, 2011).

\section{Considerações finais}

Encontramos nessa revisão predomínio de trabalhos onde foi utilizada, isoladamente, a escala de Braden. Somente um artigo avaliou as três principais escalas comparativamente com relação a sua validade preditiva, sendo encontrado um melhor desempenho da escala de Wartelow em um grupo específico de pacientes. São necessários outros estudos que correlacione as diversas escalas de avaliação de risco para UPP em pacientes críticos, para que se possa determinar com precisão o melhor instrumento para avaliação dessas feridas.

Identificamos, em vários trabalhos, o papel da equipe de enfermagem na avaliação e prevenção das UPP, recomendando a adoção de escalas de avaliação de risco, onde são identificados os pacientes e os principais fatores predisponentes para o surgimento dessas lesões. A maioria das intervenções preventivas refere-se à mudança de decúbito e uso de superfícies de apoio para alívio da pressão. Dessa forma, desconsidera-se a causa multifatorial do surgimento dessas feridas e o papel multidisciplinar, necessário na intervenção e na conduta frente ao paciente crítico em risco de UPP.

Este artigo apresenta limitações, principalmente pela perda de muitos trabalhos na seleção da amostra, por não estarem disponíveis na íntegra. Sugerem-se novos estudos de revisão que contemplem essa perda, além de novos trabalhos primários que incluíam outras escalas de avaliação, não analisadas em pacientes sujeitos a cuidados intensivos.

\section{Referências}

ARAujO, T. M.; ARAUjO, M. F. M.; CAETANO, J. A. Comparação de escalas de avaliação de risco para úlcera por pressão em pacientes em estado crítico. Acta Paulista de Enfermagem, São Paulo, v. 24, n. 5, p. 695-70, set./ out. 2011. doi: 10.1590/S0103-21002011000500016.

BAUMGARTEN, M. et al. Extrinsic risk factors for pressure ulcers early in the hospital stay: a nested case-control study. The Journals Gerontology, Oxford, v. 63, n. 4, p. 403-13, apr. 2008.

COX, J. Predictors of pressure ulcers in adult critical care patients. American Journal of Critical Care, Aliso Viejo, v. 20, n. 5, p. 364-75, sep. 2011. doi: 10.4037/ajcc2011934.

CREMASCO, M. F. et al. Úlcera por pressão: risco e gravidade do paciente e carga de trabalho de enfermagem. Acta Paulista de Enfermagem, São Paulo, v. 22, n. especial, p. 897-902, dez. 2009. doi: 10.1590/S010321002009000700011.

FERNANDES, N. C. S. Úlceras de pressão: um estudo com pacientes de unidade de terapia intensiva. 2005. 139 f. Dissertação (Mestrado) - Universidade Federal do Rio Grande do Norte, Rio Grande do Norte, 2005.

GOMES, F. S. L. et al. Fatores associados à úlcera por pressão em pacientes internados nos Centros de Terapia Intensiva de Adultos. Revista Escola de Enfermagem da USP, São Paulo, v. 44, n. 4, p. 1070-1076, dez. 2010. doi: 10.1590/S0080-62342010000400031.

HINOJOSA, I. J.; MOREIRA, D. G.; GUERRA, H. R. Beneficio de la aplicación de la escala de Norton en pacientes graves. Revista Médica Electrónica, Matanzas, v. 32, n. 5, p. 1-6, set./out. 2010.

HYUN, S. et al. Predictive validity of the braden scale for patients in intensive care units. American Journal Critical Care, Aliso Viejo, v. 22, n. 6, p. 514-520, nov. 2013. doi: 10.4037/ajcc2013991.

INSTITUTE FOR HEALTHCARE IMPROVEMENT. How to guide: prevent pressure ulcers. 2011. Available in: <http://www.ihi.org/resources/Pages/Tools/HowtoGuidePreventPressureUlcers.aspx>. Access in: 20 nov. 2016. 
LYRA, C. R. S. et al. Feridas: fundamentos e atualizações em enfermagem. 3. ed. São Caetano do Sul: Yendis, 2011.

MILTON, K. Primary care trust: critical appraisal skills programme (CASP): making sense of evidence. London. 2002. Disponível em: <http://www.casp-uk.net/>. Acesso em: 16 maio 2015.

NATIONAL PRESSURE ULCER ADVISORY PANEL. Updated staging system: pressure ulcer stages revised by NPUAP. Washington. 2016. Disponível em: <http://www. npuap.org/pr2.htm>. Acesso em: 06 maio 2015.

ROCHA, J. A.; MIRANDA M. J.; ANDRADE M. J. Abordagem terapêutica das úlceras de pressão: intervenções baseadas na evidência. Acta Médica Portuguesa, Lisboa, v. 19, n. 1, p. 29-38, jan./fev. 2006.

ROGENSKI, N. M. B.; KURCGANT, P. Avaliação da concordância na aplicação da escala de Braden interobservadores. Acta Paulista Enfermagem, São Paulo, v. 25, n. 1, p. 24-28, jan./fev. 2012a. doi: 10.1590/S010321002012000100005.

ROGENSKI, N. M. B.; KURCGANT, P. Incidência de úlceras por pressão após a implementação de um protocolo de prevenção. Revista Latino Americana Enfermagem, Ribeirão Preto, v. 20, n. 2, p. 1-7, mar./abr. 2012b. doi: 10.1590/S0104-11692012000200016.

SERPA, L. F. et al. Validade preditiva da escala de Braden para o risco de desenvolvimento de úlcera por pressão, em pacientes críticos. Revista Latino-Americana Enfermagem, Ribeirão Preto, v. 19, n. 1, p. 7-8, jan./fev. 2011. doi: 10.1590/S0104-11692011000100008.

SHAHIN, E. S. M.; DASSEN, T.; HALFENS, R. J. G. Incidence, prevention and treatment of pressure ulcers in intensive care patients: a longitudinal study. International Journal of Nursing Studies, Oxford, v. 46, n. 4, p. 413421, apr. 2009. doi: 10.1016/j.ijnurstu.2008.02.011.

SIMAO, C. M. F.; CALIRI, M. H. L.; SANTOS, C. B. Concordância entre enfermeiros quanto ao risco dos pacientes para úlcera por pressão. Acta Paulista Enfermagem, São Paulo, v. 26, n. 1, p. 30-35, jan./fev. 2013. doi: 10.1590/ S0103-21002013000100006.
SIMOES, C. E. M. S et al. Úlcera por pressão: análise de custo. In: ENCONTRO LATINO AMERICANO DE INICIAÇÃO CIENTIFICA, 14.; ENCONTRO LATINO AMERICANO DE PÓS-GRADUAÇÃO, 10., 2010, Paraíba. Anais... Paraíba: Universidade do Vale do Paraíba, 2010. p. 1-4.

SOARES, C. B. et al. Revisão integrativa: conceitos e métodos utilizados na enfermagem. Revista da Escola de Enfermagem da USP, São Paulo, v. 48, n. 2, p. 335345, abr. 2014.doi: 10.1590/S0080-6234201400002000020.

SOUZA, M. T.; SILVA, M. D.; CARVALHO, R. Revisão integrativa: o que é e como fazer. Einstein, São Paulo, v. 8, n. 1, p. 102-106, jan./mar. 2010. doi: 10.1590/S167945082010RW1134.

YEPES, D. et al. Incidencia y factores de riesgo em relación con las úlceras por presión en enfermos críticos. Medicina Intensiva, Barcelona, v. 33, n. 6, p. 276-281, ago./ set. 2009. doi: 10.1016/S0210-5691(09)72195-3.

\section{Apêndice A}

1. Artigo repetido?

( ) Sim ( ) Não

2. O estudo trata do objeto proposto para a revisão da literatura?

( ) Sim ( ) Não

3. O objetivo responde a questão norteadora do estudo?

( ) Sim ( ) Não

\section{Apêndice B}

1. Artigo original ou primário?

( ) Sim ( ) Não

2. Período de publicação atende o critério estabelecido para revisão?

( ) Sim ( ) Não

3. Idioma de publicação: inglês, português ou espanhol?

( ) Sim ( ) Não

4. Apresenta características da amostra?

( ) Sim ( ) Não

5. Apresenta país e local da coleta?

( ) Sim ( ) Não

6. O estudo apresenta dados insuficientes para a análise?

( ) Sim ( ) Não 
Critical Appraisal Skills Programme (CASP) ${ }^{1}$

\section{Anexo 1}

\begin{tabular}{|c|c|c|}
\hline Questões & Considerações & Respostas \\
\hline 1) Objetivo está claro e justificado? & $\begin{array}{l}\text { () explica objetivo } \\
\text { ( ) explica relevância do estudo } \\
\text { Comentários: }\end{array}$ & $\begin{array}{l}\text { () Sim } \\
\text { () Não }\end{array}$ \\
\hline $\begin{array}{l}\text { 2) Há adequação do desenho } \\
\text { metodológico? }\end{array}$ & $\begin{array}{l}\text { () há coerência entre os objetivos e o desenho } \\
\text { metodológico } \\
\text { Comentários: }\end{array}$ & $\begin{array}{l}\text { () Sim } \\
\text { ( ) Não }\end{array}$ \\
\hline $\begin{array}{l}\text { 3) os procedimentos teórico } \\
\text { metodológicos são apresentados e } \\
\text { discutidos? }\end{array}$ & $\begin{array}{l}\text { () há justificativa da escolha do referencial, método } \\
\text { () explica os procedimentos metodológicos } \\
\text { Comentários: }\end{array}$ & $\begin{array}{l}\text { () Sim } \\
\text { () Não }\end{array}$ \\
\hline $\begin{array}{l}\text { 4) A amostra de estudo foi } \\
\text { selecionada adequadamente? }\end{array}$ & $\begin{array}{l}\text { ( ) explica os critérios de seleção (inclusão e exclusão) da } \\
\text { amostra de estudo. } \\
\text { Comentários: }\end{array}$ & $\begin{array}{l}\text { () Sim } \\
\text { ( ) Não }\end{array}$ \\
\hline 5) A coleta de dados está detalhada? & $\begin{array}{l}\text { ( ) explica a forma de coleta de dados (entrevista, grupo } \\
\text { focal, ...) } \\
\text { ( ) explica o uso de instrumento para a coleta (questionário, } \\
\text { roteiro, ...) } \\
\text { Comentários: }\end{array}$ & $\begin{array}{l}\text { () Sim } \\
\text { ( ) Não }\end{array}$ \\
\hline $\begin{array}{l}\text { 6) A relação entre pesquisador e } \\
\text { pesquisados foi considerada? }\end{array}$ & $\begin{array}{l}\text { ( ) o examinador examina criticamente a sua atuação como } \\
\text { pesquisador, reconhecendo potencial de viés (na seleção } \\
\text { da amostra, na formulação de perguntas) } \\
\text { ( ) descreve ajustes e suas implicações no desenho da } \\
\text { pesquisa } \\
\text { Comentários: }\end{array}$ & $\begin{array}{l}\text { () Sim } \\
\text { () Não }\end{array}$ \\
\hline $\begin{array}{l}\text { 7) os aspectos éticos de uma } \\
\text { pesquisa foram respeitados? }\end{array}$ & $\begin{array}{l}\text { () há menção de aprovação por comitê de ética } \\
\text { ( ) há menção do termo de consentimento autorizado } \\
\text { Comentários: }\end{array}$ & $\begin{array}{l}\text { ( ) Sim } \\
\text { ( ) Não }\end{array}$ \\
\hline $\begin{array}{l}\text { 8) A análise de dados é rigorosa e } \\
\text { fundamentada? } \\
\text { Especifica os testes estatísticos? }\end{array}$ & $\begin{array}{l}\text { () explica o processo de análise } \\
\text { ( ) explica como as categorias de análise foram identificadas } \\
\text { () os resultados refletem os achados } \\
\text { Comentários: }\end{array}$ & $\begin{array}{l}\text { () Sim } \\
\text { ( ) Não }\end{array}$ \\
\hline $\begin{array}{l}\text { 9) Resultados são apresentados e } \\
\text { discutidos com prioridade? }\end{array}$ & $\begin{array}{l}\text { ( ) explica os resultados ( ) dialoga seus resultados com o de } \\
\text { outros pesquisadores } \\
\text { ( ) os resultados são analisados à luz da questão do estudo } \\
\text { Comentários: }\end{array}$ & $\begin{array}{l}\text { () Sim } \\
\text { () Não }\end{array}$ \\
\hline 10) Qual o valor da pesquisa? & $\begin{array}{l}\text { ( ) explica a contribuição e limitações da pesquisa (para a } \\
\text { pratica, construção do conhecimento, ...) } \\
\text { ( ) indica novas questões de pesquisa } \\
\text { Comentários: }\end{array}$ & $\begin{array}{l}\text { () Sim } \\
\text { () Não }\end{array}$ \\
\hline
\end{tabular}

Fonte: 1-Adaptado de Critical Appraisal Skills Programme (CASP) - Programa de habilidades em leitura crítica, 2002.

Resultado: () Nível A - 6-10 () Nível B - até 5 